Fixed Point Theory, 20(2019), No. 1, 59-70

DOI: $10.24193 /$ fpt-ro.2019.1.04

http://www.math.ubbcluj.ro/ nodeacj/sfptcj.html

\title{
MAIA TYPE FIXED POINT THEOREMS FOR PREŠIĆ TYPE OPERATORS
}

\author{
MARGARETA-ELIZA BALAZS \\ Technical University of Cluj-Napoca, North University Center of Baia Mare \\ 76 Victoriei st., Baia Mare, 430122, Romania \\ E-mail: elizabalazs8@gmail.com
}

\begin{abstract}
In this paper, we extend to the case of product spaces, two generalizations of Maia fixed point theorem [Maia, Maria Grazia. Un'osservazione sulle contrazioni metriche. (Italian) Rend. Sem. Mat. Univ. Padova 401968 139-143] given by Rus I. A. in [Rus, Ioan A. Generalized contractions. Seminar on Fixed Point Theory, Babeş Bolyai Univ., Cluj-Napoca, 1983, Preprint nr. 3, pp. 1-130, 35] and [Rus, Ioan A. Basic problem for Maia's theorem. Seminar on Fixed Point Theory, Babeş Bolyai Univ., Cluj-Napoca, 1981, Preprint nr. 3, pp. 112-115]. Following the results in [Petruşel, A., Fredholm-Volterra integral equations and Maia's theorem, Seminar on Fixed Point Theory, Babes Bolyai Univ., Cluj-Napoca, (1988), Preprint nr. 3, pp. 79-82], a theorem on the existence and uniqueness of solutions of Fredholm-Volterra integral equations, using a theorem of Maia type in product metric spaces, is proved.
\end{abstract}

Key Words and Phrases: Fixed point, Maia, Prešić type contraction, two metrics.

2010 Mathematics Subject Classification: 54H25, 47H10.

\section{REFERENCES}

[1] M.E. Balazs, A Maia type fixed point theorem for Prešić-Kannan operators, Miskolc. Math. Notes, 18(2017), no. 1, 71-81.

[2] S. Banach, Sur les óperations dans les ensembles abstraits et leur application aux équations intégrales, Fund. Math., 3(1922), 133-181.

[3] L.B. Ćirić, S.B. Prešić, On Prešić type generalization of the Banach contraction mapping principle, Acta Math. Univ. Comen. (N.S.), 76(2007), no. 2, 143-147.

[4] M.G. Maia, Un'osservazione sulle contrazioni metriche, (Italian), Rend. Sem. Mat. Univ. Padova, 40(1968), 139-143.

[5] M. Păcurar, Approximating common fixed points of Prešić-Kannan type operators by a multistep iterative method, An. Ştiinţ. Univ. "Ovidius" Constanţa, Ser. Mat., 17(2009), no. 1, 153168.

[6] M. Păcurar, Iterative Methods for Fixed Point Approximation, Risoprint, 2009.

[7] M. Păcurar, A multi-step iterative method for approximating fixed points of Prešić-Kannan operators, Acta Math. Univ. Comen. (N.S.), 79(2010), no. 1, 77-88.

[8] A. Petruşel, Fredholm-Volterra integral equations and Maia's theorem, Seminar on Fixed Point Theory, Babeş Bolyai Univ., Cluj-Napoca, 1988, Preprint nr. 3, 79-82.

[9] A. Petruşel, I.A. Rus, Fixed point theory for multivalued operators on a set with two metrics, Fixed Point Theory, 8(2007), 97-104. 
[10] S.B. Prešić, Sur une classe d'inéquations aux différences finies et sur la convergence de certaines suites, (French), Publ. Inst. Math. (Beograd) (N.S.), 5(19)(1965), 75-78.

[11] I.A. Rus, Metrical fixed point theorems, Univ. of Cluj-Napoca, Cluj-Napoca, 1979, 23-34.

[12] I.A. Rus, Generalized contractions, Seminar on Fixed Point Theory, Babeş Bolyai Univ., ClujNapoca, 1983, Preprint nr. 3, 1-130.

[13] I.A. Rus, Basic problem for Maia's theorem, Seminar on Fixed Point Theory, Babeş Bolyai Univ., Cluj-Napoca, 1981, Preprint nr. 3, 112-115.

[14] I.A. Rus, An abstract point of view in the nonlinear difference equations, Conf. Annal., Functional Equations Appl. and Convexity, Cluj-Napoca, 1999, 272-276.

[15] I.A. Rus, Data dependence of the fixed points in a set with two metrics, Fixed Point Theory, 8(2007), 115-123.

[16] I.A. Rus, A. Petruşel, G. Petruşel, Fixed Point Theory, Cluj University Press, 2008.

Received: June 7, 2017; Accepted: October 1st, $201 \%$. 